Research Article

\title{
Implications of Regionalizing Care in the Developing World: Impact of Distance to Referral Center on Compliance to Biopsy Recommendations in a Brazilian Prostate Cancer Screening Cohort
}

\author{
Alexis R. Freedland $\left(\mathbb{D},{ }^{1}\right.$ Roberto L. Muller $\mathbb{D}^{2},{ }^{2}$ Cathrine Hoyo ${ }^{(D)},{ }^{3}$ Elizabeth L. Turner, ${ }^{4,5}$ \\ Patricia G. Moorman $\mathbb{D}^{6},{ }^{6}$ Eliney F. Faria $\mathbb{D}^{7},{ }^{7}$ Gustavo F. Carvalhal $\mathbb{D}^{8},{ }^{8}$ Rodolfo B. Reis $\mathbb{D}^{9}$, \\ Edmundo C. Mauad, ${ }^{10}$ Andre L. Carvalho, ${ }^{8}$ and Stephen J. Freedland $\mathbb{D i D}^{11}$ \\ ${ }^{1}$ Department of Epidemiology, UCI School of Medicine, University of California, Irvine, CA, USA \\ ${ }^{2}$ Division of Urology, Center of Oncologic Research, Florianopolis, Santa Catarina, Brazil \\ ${ }^{3}$ Department of Biological Sciences, North Carolina State University, Raleigh, NC, USA \\ ${ }^{4}$ Global Health Institute, Duke University, Durham, NC, USA \\ ${ }^{5}$ Department of Biostatistics and Bioinformatics, Duke University Graduate School, Durham, NC, USA \\ ${ }^{6}$ Department of Community and Family Medicine, Cancer Control and Population Sciences, Duke Cancer Institute, \\ Durham, NC, USA \\ ${ }^{7}$ Division of Urologic Oncology and Laparoscopy, Barretos Cancer Hospital, Barretos, São Paulo, Brazil \\ ${ }^{8}$ Research Support Center, Barretos Cancer Hospital, Barretos, São Paulo, Brazil \\ ${ }^{9}$ Division of Urology, Ribeirao Preto Medical School of Sao Paulo University (USP), Ribeirao Preto, São Paulo, Brazil \\ ${ }^{10}$ Department of Preventative Medicine, Barretos Cancer Hospital and Pio XII Foundation, Barretos, São Paulo, Brazil \\ ${ }^{11}$ Division of Urology, Department of Surgery, Cedars-Sinai Medical Center, Los Angeles, CA, USA \\ Correspondence should be addressed to Alexis R. Freedland; gainesar@uci.edu
}

Received 12 March 2021; Accepted 9 June 2021; Published 22 June 2021

Academic Editor: Hendrik Van Poppel

Copyright (c) 2021 Alexis R. Freedland et al. This is an open access article distributed under the Creative Commons Attribution License, which permits unrestricted use, distribution, and reproduction in any medium, provided the original work is properly cited.

Given growing specialization in medical care, optimal care may require regionalization, which may create access barriers. We tested this within a large prostate cancer (PC) screening program in Brazil. In 2004-2007, Barretos Cancer Hospital prospectively screened men for PC throughout rural Brazil. Men with abnormal screen were referred for follow-up and possible biopsy. We tested the link between distance from screening site to Barretos Cancer Hospital and risk of noncompliance with showing up for biopsy, PC on biopsy and, among those with PC, PC grade using crude and multivariable logistic regression analysis. Among 10,467 men undergoing initial screen, median distance was $257 \mathrm{~km}$ (IQR: $135-718 \mathrm{~km}$ ). On crude and multivariable analyses, farther distance was significantly linked with biopsy noncompliance $(\mathrm{OR} / 100 \mathrm{~km}: 0.83, P<0.001)$. Among men who lived within $150 \mathrm{~km}$ of Barretos Cancer Hospital, distance was unrelated to compliance (OR/100 km: 1.09, $P=0.87)$. There was no association between distance and PC risk or PC grade (all $P>0.25$ ). In Brazil, where distances to referral centers can be large, greater distance was related to reduced biopsy compliance in a PC screening cohort. Among men who lived within $150 \mathrm{~km}$, distance was unrelated to compliance. Care regionalization may reduce access when distances are large. 


\section{Introduction}

A 2011 study of overall cancer mortality in Brazilian state capitals (urban areas) vs. outlying municipalities (rural areas) from 1980 to 2006 found a mortality increase of more than $100 \%$ for all cancer types [1]. While prostate cancer (PC) mortality increased over time in all regions, mortality increased faster among men in rural areas. Although the exact reasons for this increase are unclear, it is conceivable that rural men have reduced access to healthcare and thus do not receive the same benefits of early detection and treatment.

Given the growing number of aging people with varied health needs and growing specialization in medical care [2-4], there is increasing realization that optimal care for certain procedures may require regionalization $[5,6]$. While this comes with the benefit of creating high-volume centers to drive optimal outcomes, this also comes at the cost of increased burden to travel, including obtaining transportation [7], loss of wages, and being away from home $[6,8]$. A 2016 multinational systematic review of 108 studies from developed countries evaluated the association between distance to healthcare services and subject outcomes and found some studies showed greater distances negatively impacted access to care [9]. Importantly, the distances were typically $\leq 100$ miles. Given the large distances needed to travel for care in Brazil, there is concern that subjects may not follow up for required care [10]. It is likely the distance subjects are willing to travel depends on the perceived benefits of receiving such specialized care. As such, the nature of the medical condition and the distance to the care facility may dictate whether the benefits of regionalization outweigh the barriers this creates for access.

The 2004-2007 Barretos Cancer Hospital (BCH) medical Mobile Cancer Prevention Screening Unit (MCPSU) is the largest PC screening study in South America [11]. Due to its study design, wherein men were initially screened in local communities and those with abnormal screens were referred to a care facility often hundreds of kilometers $(\mathrm{km})$ away, the MCPSU provides a unique opportunity to study the impact of care regionalization on PC in Brazil. We hypothesized greater distance will have a negative impact on receipt of follow-up care after PC screening and only those with the highest PC risk will adhere due to greater perceived benefits of care, leading to a positive link between distance and aggressive PC among those who adhere.

\section{Materials and Methods}

2.1. Data Collection. After obtaining institutional review board approval, we conducted a cross-sectional study of 11,117 men who underwent initial PC screening as a part of the MCPSU from 2004 to 2007 in 231 cities in Southern Brazil. Because these areas had limited access to health services, PC screening took place on BCH MCPSUs. Screening was targeted to specific low socioeconomic status (SES) cities. Distances between local screening sites and regional diagnostic and treatment facilities at $\mathrm{BCH}$ were estimated using the shortest route obtained between each participating city and the address of $\mathrm{BCH}$ calculated by Google Maps (Google LLC, Mountain View, CA, USA).

Enrollment methods were previously described $[11,12]$ and informed consent was obtained from each participant. Participants received free PC screens from MCPSU personnel using on-site prostate specific antigen (PSA) testing and digital rectal exams (DRE). The same staff performed PSAs and DREs at every site. Men with total PSA (tPSA) $>4.0 \mathrm{ng} / \mathrm{mL}$, tPSA $2.5-4.0 \mathrm{ng} / \mathrm{mL}$ with percent-free PSA (pfPSA) $\leq 15 \%$, or DRE suspicious of PC were contacted by mail or phone and referred to $\mathrm{BCH}$ for follow-up for possible biopsy. Men were required to follow up within six months after screen. Once at $\mathrm{BCH}$, repeat PSA and DRE were performed. Men whose indications were confirmed were recommended to undergo biopsy.

2.2. Study Participants. Among 11,117 men undergoing initial screen, we excluded men missing data on education $(n=6)$ or PSA $(n=644)$, leaving $10,467(94 \%)$ men. Of these, $1,561(15 \%)$ were referred for further evaluation, of which $1,131(72 \%)$ complied and $430(28 \%)$ did not. Of the 1,131 men who presented to $\mathrm{BCH}$, all underwent a confirmation screening. 273 had normal screenings and were no longer recommended for biopsy and eight were excluded from analysis due to inconclusive biopsy pathology. Therefore, for secondary analyses, we examined 850 men for PC on biopsy and PC grade (Figure 1).

2.3. Statistical Analysis. Associations between clinical or demographic factors for compliant and noncompliant men were evaluated using a two-sample $t$-test with equal variances for normally distributed continuous variables, Wilcoxon rank-sum test for nonnormally distributed continuously variables, and Pearson's chi-square test for categorical variables. In multivariable analyses, nonnormally distributed variables were logarithmically transformed to obtain normal distribution of the data. Variables ascertained via questionnaire included age (continuous, years), initial screening tPSA (continuous, logarithmically transformed), distance from screening site to $\mathrm{BCH}$ (categorical, $<250$, $250-500,500-1000, \geq 1000 \mathrm{~km}$, and logarithmically transformed continuous), education level (categorical, illiterate, incomplete primary, complete primary, high school, and college), PC family history (yes/no), family history of other cancers (yes/no), year of screening (categorical, 2004, 2005, 2006, and 2007), and DRE findings (normal/abnormal). Primary analyses used distance as a continuous logarithmically transformed variable. To provide clinical context, we also examined distance categorically in $250 \mathrm{~km}$ categories. Logistic regression was used to test the association between distance and biopsy noncompliance. Multivariable models were adjusted for the aforementioned covariates. We used a locally weighted smoothed scatterplot (LOWESS) to graphically depict the association between distance and biopsy compliance. Based upon visual review of the LOWESS, a post hoc subset analysis was conducted testing the association between distance and compliance among men with shorter distances ( $150 \mathrm{~km}$ cutoff). 


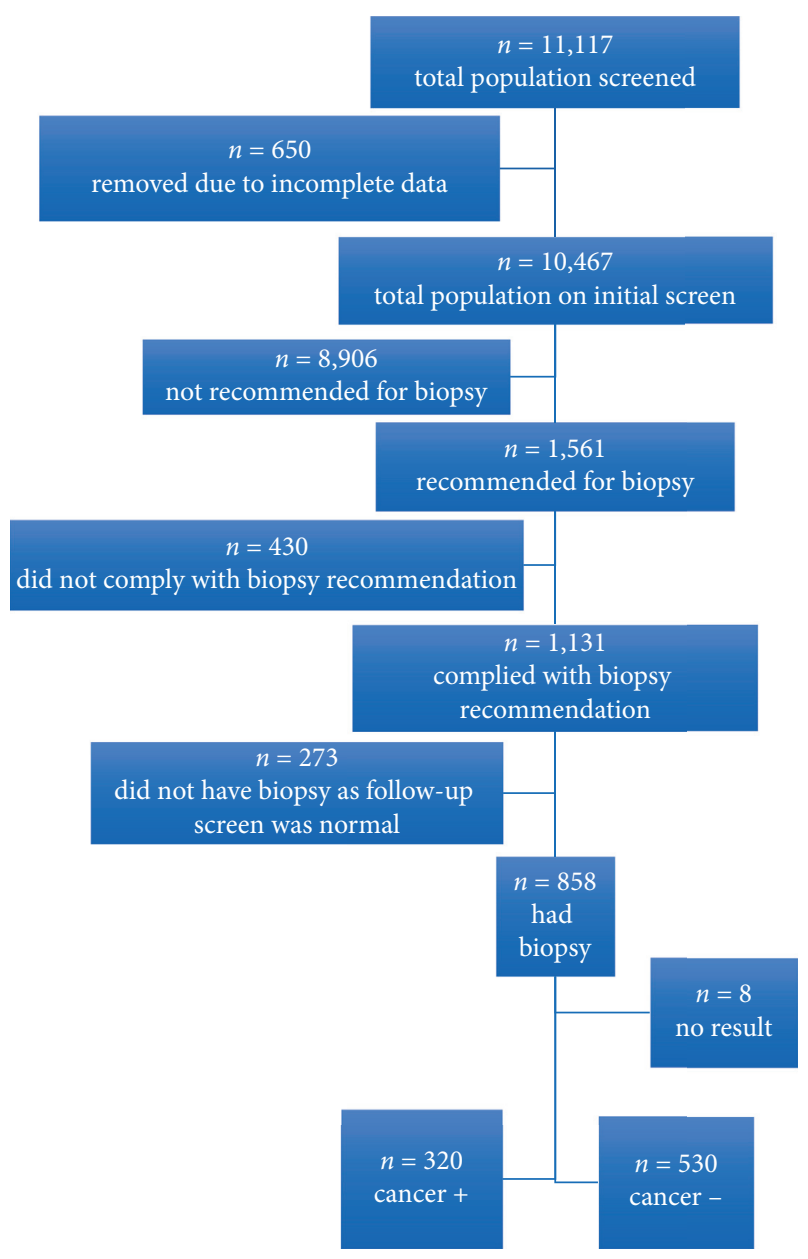

Figure 1: Consort flow diagram describing the progression of subjects through the study.

Secondary analyses evaluated the association between distance and PC risk on biopsy using crude and multivariable models, adjusting for the aforementioned covariates. Finally, we used multinomial logistic regression to assess the association between distance and PC grade defined as no PC (referent group), low-grade PC (Grade Group 1), and highgrade PC (Grade Group $\geq 2$ ). [13] All statistical analyses were performed using Stata 13.1 (StataCorp, College Station, TX, USA). Two-tailed $P$ values of $\leq 0.05$ were considered statistically significant.

\section{Results}

3.1. Baseline Clinical Characteristics and Cancer Details among Compliant Men. Noncompliant men $(n=430,27.5 \%)$ were older at screen (mean: 68 vs. 66 years, $P<0.001$ ), had higher enrollment tPSA ( 4.9 vs. $4.2 \mathrm{ng} / \mathrm{mL}$, $P<0.001)$, and were less likely to have an abnormal DRE $(19.5 \%$ vs. $33.4 \%, P<0.001)$ than compliant men $(n=1,131$, $72.5 \%)$. Noncompliant men had less education (illiterate + incomplete primary school: $91.7 \%$ vs. $89.2 \%, P<0.001$ ) and were more likely to live farther away from $\mathrm{BCH}$ (median: 921 vs. $225 \mathrm{~km}, P<0.001$ ). There were no statistically significant differences in family history for any cancer $(P=0.07)$ or PC $(P=0.15)$ by compliance status (Table 1$)$.

Of the 850 compliant men who underwent a biopsy, 320 (37.7\%) had PC, of which $65.1 \%$ had low-grade PC (Grade Group 1) and most had Stage 1 disease (74.5\%). The median PSA of men on biopsy was $7 \mathrm{ng} / \mathrm{mL}$ (IQR: $4-16 \mathrm{ng} / \mathrm{mL}$ ) and the median number of positive cores was 3/12 (IQR: 2-5, Table 1).

3.2. Distance and Noncompliance. On crude analysis, noncompliant men lived further from BCH $(P<0.001)$. Specifically, median (IQR) distance for compliant men was $225 \mathrm{~km}(119-414 \mathrm{~km})$ vs. $921 \mathrm{~km}(333-1603 \mathrm{~km})$ for noncompliant men. To put this in a more clinically useful form, we also evaluated distance in $250 \mathrm{~km}$ categories and noted that, relative to men who lived $<250 \mathrm{~km}$ from $\mathrm{BCH}$, those who lived farther away were more likely to be noncompliant with follow-up recommendations $(250-500 \mathrm{~km}$ : OR: 2.00 , 95\% CI: $1.40-2.85 ; \quad 500-1000 \mathrm{~km}:$ OR: $5.88, \quad 95 \%$ CI: $4.07-8.51$; $\geq 1000 \mathrm{~km}$ : OR: $15.98,95 \%$ CI: $11.41-22.38$, $P<0.001$; Table 2). After adjusting for clinical and demographic factors, results were largely unchanged (Table 2).

Figure 2 shows the relationship between distance and compliance. Compliance declines linearly with distance until distance reaches $\sim 1,500 \mathrm{~km}$, after which numbers are small, and the beginning of the association had a less steep decline. To explore the earlier part of the curve, we chose a distance of $150 \mathrm{~km}$ (chosen post hoc) and examined, within this limited range, whether distance impacted compliance. On multivariable analysis with distance as a continuous variable, distance was unrelated to compliance when examining men who lived within $150 \mathrm{~km}$ of BCH (OR/100 km: 1.09, 95\% CI: $0.40-2.91, P=0.87$ ).

3.3. Distance and Biopsy Outcomes. On crude analysis, there was no statistically significant association between distance and PC on biopsy $(P=0.45)$ as well as both low-grade PC $(P=0.56)$ and high-grade PC $(P=0.50$, Table 3$)$. Results were unchanged on multivariable analysis for overall PC risk $(P=0.80)$ and low $(P=0.79)$ and high-grade disease $(P=0.94)$.

\section{Discussion}

There is increased interest in understanding the impact of regionalizing care on access. The impact of greater distance on receipt of follow-up care after PC screening in a developing country like Brazil, where large proportions of the population reside in rural areas, is unknown. We tested if there was a link between distance and compliance to followup recommendations or being diagnosed with aggressive PC among men who underwent initial PC screening in their local community. We found that longer distance was significantly associated with follow-up noncompliance (OR/ $100 \mathrm{~km}$ : 0.83, $P<0.001)$. However, among men who lived within $150 \mathrm{~km}$ of the referral center, distance was unrelated to compliance $(\mathrm{OR} / 100 \mathrm{~km}: 1.09, P=0.87)$. We found no association between distance and PC risk or PC grade on 
TABLE 1: Baseline characteristics of men undergoing initial PC screening by Brazilian mobile medical units stratified by compliance to biopsy recommendation $(n=1,561)$ and PC details among compliant men who underwent biopsy $(n=318)$.

\begin{tabular}{|c|c|c|c|}
\hline \multirow{3}{*}{ Baseline characteristics } & \multirow{3}{*}{$\begin{array}{l}\text { Compliant men } \\
N=1,131(72.5)\end{array}$} & \\
\hline & & Noncompliant men & \multirow[t]{2}{*}{$P^{+}$} \\
\hline & & $N=430(27.5)$ & \\
\hline \multicolumn{4}{|l|}{ Educational level attained } \\
\hline Illiterate & $279(24.7)$ & $165(38.4)$ & \multirow{5}{*}{$<0.001$} \\
\hline Incomplete primary & $729(64.5)$ & $229(53.3)$ & \\
\hline Complete primary & $86(7.6)$ & $17(4.0)$ & \\
\hline High school & $30(2.7)$ & $15(3.5)$ & \\
\hline College & $7(0.6)$ & $4(0.9)$ & \\
\hline \multicolumn{4}{|l|}{ Year of screening } \\
\hline 2004 & $230(20.3)$ & $82(19.1)$ & \multirow{4}{*}{$<0.001$} \\
\hline 2005 & $355(31.4)$ & $80(18.6)$ & \\
\hline 2006 & $333(29.4)$ & $102(23.7)$ & \\
\hline 2007 & $213(18.8)$ & $166(38.6)$ & \\
\hline \multicolumn{4}{|l|}{ Positive family history } \\
\hline $\mathrm{PC}$ & $56(5.0)$ & $14(3.3)$ & 0.15 \\
\hline Any cancer & $122(10.8)$ & $33(7.7)$ & 0.07 \\
\hline DRE suspicious of PC & $378(33.4)$ & $84(19.5)$ & $<0.001$ \\
\hline \multicolumn{4}{|l|}{ Cancer status } \\
\hline Positive & $320(37.7)$ & - & - \\
\hline Negative & $530(62.4)$ & - & - \\
\hline \multicolumn{4}{|l|}{ Grade group } \\
\hline I (Gleason 2-6) & $207(65.1)$ & - & - \\
\hline II-III (Gleason 7) & $85(26.7)$ & - & - \\
\hline IV-V (Gleason 8-10) & $26(8.2)$ & - & - \\
\hline \multicolumn{4}{|l|}{ Clinical stage } \\
\hline Stage I & $237(74.5)$ & - & - \\
\hline Stage II & $42(13.2)$ & - & - \\
\hline Stage III & $16(5.0)$ & - & - \\
\hline \multirow[t]{2}{*}{ Stage IV } & $23(7.2)$ & - & - \\
\hline & \multicolumn{2}{|c|}{ Mean (SD) } & $P^{*}$ \\
\hline \multirow[t]{2}{*}{ Age (years) } & $66(9.0)$ & $68(9.3)$ & $<0.001$ \\
\hline & \multicolumn{2}{|c|}{ Median (IQR) } & $P^{\#}$ \\
\hline tPSA $(\mathrm{ng} / \mathrm{mL})$ & $4.2(2.6-7.1)$ & $4.9(3.5-7.7)$ & $<0.001$ \\
\hline Distance from Barretos (km) & $225(119-414)$ & $921(333-1603)$ & $<0.001$ \\
\hline Total number of positive cores ${ }^{@}$ & $3(2-5)$ & - & - \\
\hline Total number of cores taken ${ }^{@}$ & $12(10-14)$ & - & - \\
\hline Prostate volume (cc)@ & $33(25-46)$ & - & - \\
\hline tPSA (ng/mL) ${ }^{@}$ & $7(4-16)$ & - & - \\
\hline
\end{tabular}

Statistical analyses: ${ }^{*} t$-test; ${ }^{+}$chi-squared test; ${ }^{*}$ rank-sum. DRE: digital rectal exam; IQR: interquartile range; km: kilometer; $P: P$ value; PC: prostate cancer; tPSA: total serum prostate specific antigen $(\mathrm{ng} / \mathrm{mL}) .{ }^{\circledR}$ Among 318 men with available data.

TABLE 2: Association between distance and risk of noncompliance.

\begin{tabular}{|c|c|c|c|c|c|}
\hline Model: logistic regression & Distance variable format & Distance $(\mathrm{km})$ & OR & $95 \% \mathrm{CI}$ & $P$ value \\
\hline \multirow{4}{*}{ Unadjusted } & Continuous & - & 2.81 & $2.48-3.19$ & $<0.001$ \\
\hline & & $250-499$ & 2.00 & $1.40-2.85$ & \\
\hline & Categorical relative to $0-249 \mathrm{~km}$ & $500-999$ & 5.88 & $4.07-8.51$ & $<0.001$ \\
\hline & & $\geq 1000$ & 15.98 & $11.41-22.38$ & \\
\hline \multirow{4}{*}{ Multivariable } & Continuous & - & 2.71 & $2.37-3.09$ & $<0.001$ \\
\hline & & $250-499$ & 1.87 & $1.30-2.70$ & \\
\hline & Categorical relative to $0-249 \mathrm{~km}$ & $500-999$ & 5.36 & $3.65-7.87$ & $<0.001$ \\
\hline & & $\geq 1000$ & 14.91 & $10.45-21.28$ & \\
\hline
\end{tabular}

Multivariable analysis adjusted for age, log PSA, education, family history of cancer, family history of PC, DRE, and screening year. CI: confidence interval; $\mathrm{km}$ : kilometers; OR: odds ratio. 


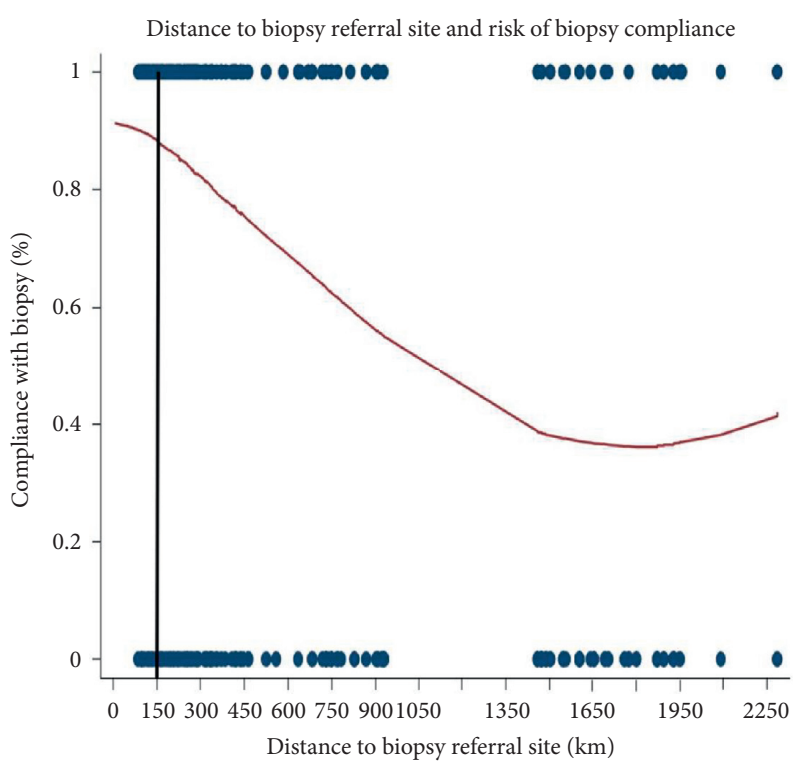

FIGURE 2: LOWESS smoothened curve evaluating the univariable association between distance to biopsy referral site and compliance.

TABLE 3: The association between distance and risk of PC and PC grade $(n=850)$.

\begin{tabular}{|c|c|c|c|c|c|}
\hline \multicolumn{6}{|c|}{ Association between distance and risk of PC } \\
\hline Model: logistic regression & Distance variable format & Distance $(\mathrm{km})$ & OR & $95 \% \mathrm{CI}$ & $P$ value \\
\hline \multirow{4}{*}{ Unadjusted } & Continuous & - & 1.05 & $0.92-1.20$ & 0.45 \\
\hline & & $250-499$ & 1.05 & $0.76-1.46$ & \\
\hline & Categorical relative to $0-249 \mathrm{~km}$ & $500-999$ & 0.87 & $0.54-1.40$ & 0.37 \\
\hline & & $\geq 1000$ & 1.45 & $0.91-2.30$ & \\
\hline \multirow{4}{*}{ Multivariable } & Continuous & - & 0.98 & $0.85-1.14$ & 0.80 \\
\hline & & $250-499$ & 0.96 & $0.67-1.37$ & \\
\hline & Categorical relative to $0-249 \mathrm{~km}$ & $500-999$ & 0.69 & $0.41-1.17$ & 0.39 \\
\hline & & $\geq 1000$ & 1.22 & $0.74-2.03$ & \\
\hline \multicolumn{6}{|c|}{ Association between distance and PC grade (relative to no PC) } \\
\hline Model: multinomial logistic regression & Distance variable format & Distance $(\mathrm{km})$ & OR & $95 \% \mathrm{CI}$ & $P$ value \\
\hline \multicolumn{6}{|c|}{ Cancer outcome: low-grade PC (Grade Group I) } \\
\hline \multirow{4}{*}{ Unadjusted } & Continuous & - & 1.04 & $0.91-1.20$ & 0.56 \\
\hline & & $250-499$ & 0.97 & $0.69-1.36$ & \\
\hline & Categorical relative to $0-249 \mathrm{~km}$ & $500-999$ & 0.86 & $0.53-1.40$ & 0.27 \\
\hline & & $\geq 1000$ & 1.38 & $0.86-2.22$ & \\
\hline \multirow{4}{*}{ Multivariable } & Continuous & - & 0.98 & $0.84-1.14$ & 0.79 \\
\hline & & $250-499$ & 0.90 & $0.62-1.30$ & \\
\hline & Categorical relative to $0-249 \mathrm{~km}$ & $500-999$ & 0.69 & $0.40-1.17$ & 0.32 \\
\hline & & $\geq 1000$ & 1.20 & $0.72-2.00$ & \\
\hline \multicolumn{6}{|c|}{ Cancer outcome: high-grade PC (Grade Group $\geq 2)$} \\
\hline \multirow{4}{*}{ Unadjusted } & Continuous & - & 1.13 & $0.79-1.64$ & 0.50 \\
\hline & & $250-499$ & 2.44 & $1.03-5.79$ & \\
\hline & Categorical relative to $0-249 \mathrm{~km}$ & $500-999$ & 0.96 & $0.20-4.48$ & 0.27 \\
\hline & & $\geq 1000$ & 2.39 & $0.72-7.93$ & \\
\hline \multirow{4}{*}{ Multivariable } & Continuous & - & 0.98 & $0.65-1.49$ & 0.94 \\
\hline & & $250-499$ & 2.15 & $0.83-5.57$ & \\
\hline & Categorical relative to $0-249 \mathrm{~km}$ & $500-999$ & 0.63 & $0.12-3.33$ & 0.32 \\
\hline & & $\geq 1000$ & 1.47 & $0.34-6.41$ & \\
\hline
\end{tabular}

Multivariable analysis adjusted for age, log PSA, education, family history of cancer, family history of PC, DRE, and screening year. CI: confidence interval; km: kilometers; OR: odds ratio; PC: prostate cancer; RRR: relative risk ratio. 
biopsy (all $P>0.25$ ). Our results show that in Brazil, where distances can be very large, longer distance can negatively impact follow-up among men undergoing PC screening manifesting as a barrier to care. Whether similar associations are seen in other countries and healthcare systems requires further study.

While distance to care is a potential barrier restricting access, data evaluating the association between distance and compliance with cancer screening are limited. Though our study is the first to analyze the association between distance and PC in the developing world, few studies addressed this issue in the US [4, 14-16]. One single-center study examined the association between distance and missed clinic appointments among men suspected of or being treated for PC [14]. Among 1,341 scheduled clinic encounters for 576 patients, greater distance was associated with improved compliance (compliant median $=11.8 \mathrm{mi}$ vs. missed appointment $=10.4 \mathrm{mi}, P=0.04$ ). Investigators found that greater distance was not a significant barrier to access to healthcare, though the relatively small distances traveled in this study may not be applicable in developing countries where distances required to travel are often significantly larger $[4,15]$. Indeed, our study agrees that small distances do not create barriers for access. Our findings are in line with a study from the Prostate, Lung, Colon, and Ovarian Cancer Screening Trial, which found that subjects who lived farthest away (units unknown) were more likely to be noncompliant with adherence to first-round screening than subjects who lived closer (near: 14\%; midrange: 18\%; far: 20\%). However, the association between farther distance and poor compliance became nonsignificant on multivariable adjustment for SES, lifestyle, and demographic confounders [16]. Unfortunately, in our study, we were not able to adjust for SES defined by wealth and occupation or other lifestyle factors as these data were unavailable. Collectively, while these data suggest that large distances may be linked with poor compliance, this had never been studied in a developing country like Brazil.

To explore the barriers that regionalization can create, we tested the link between distance and risk of noncompliance with showing up to have follow-up and a possible biopsy in a population-based PC screening cohort in Brazil. We found that increased distance to referral facility was significantly associated with increased risk of noncompliance to biopsy recommendations (2-16 times more likely depending on distance). Importantly, when distances were $<150 \mathrm{~km}$, distance was unrelated to compliance. We found no association between distance and PC detection or grade on biopsy. These data suggest that while regionalization of care may in theory improve quality, it comes at the cost of reduced compliance and thus reduced access. This represents a significant barrier to optimal care if distances are large. Regarding PC screening and biopsy, our data suggest distances up to $150 \mathrm{~km}$ do not create barriers for care in Brazil. Alternative thresholds may apply for other services and in other countries.

While distance created a barrier for compliance, we hypothesized that men who stood the most to benefit from screening (i.e., those at higher risk of aggressive PC) would be more compliant creating a positive association between greater distance and more aggressive tumors. Indeed, such an association was found in breast cancer where increased distance (highest category $\geq 58 \mathrm{~km}$ ) to screening site was associated with higher stage at diagnosis $(P=0.037)$ [17]. Whether this suggests that people who live further distances only present for screening if they have higher risk disease, or whether distance-related barriers leading to delayed diagnosis and advanced disease is unknown, we did not find a relationship consistent with either hypothesis. However, it is worth noting that, among compliant men, the upper IQR for tPSAs $=7.1 \mathrm{ng} / \mathrm{mL}$, a level that is rarely associated with symptoms from advanced PC. As such, the majority of men in our study would have been asymptomatic or have minimal, less aggressive disease, though data on symptoms was unavailable. Further, men who were recommended for follow-up were not informed of their actual PSA values, so they may not have been aware of their actual PC risk. Instead, they were told that they were at "increased" risk. It is unknown whether our results would have been different if men were informed of their PC risk via receipt of actual PSA values or using a risk calculator to estimate PC risk. Indeed, others have also found distance was related to stage at diagnosis, albeit in colorectal and lung cancer [18-21]. Thus, though we found no link between distance and grade, given conflicting literature from other cancers, more research is needed on this topic. Of note, only 37\% of men in our study had PC on initial biopsy, $65 \%$ of which were Grade Group 1 PCs. Viewed alternatively, only $13 \%$ of men in our study had higher grade disease (Grade Group 2-5). Whether our results would have differed in a cohort that included a higher percent of high-risk men is unknown.

Our study has limitations. First, these data were not collected with research intent. However, as any misclassifications in the data tend to bias results to the null, it is possible that the association between distance and poor compliance may be stronger than observed. Additional information such as SES, race, body composition, and comorbidities was unavailable. How much these would have influenced our results is unknown. Additional studies are needed to understand the impact of other factors on compliance and its effect on long-term PC outcomes. The outcome of noncompliant men is unknown. Perhaps they received appropriate care elsewhere or perhaps noncompliance delayed diagnosis of their PC and led to worse outcomes. Measurements of distance are subjective: we chose distance traveled by road, though other evaluations of distance may have alternative findings. The impact of distance on received care would be influenced not only by physical distance but the infrastructure available to overcome the barriers distance presents (e.g., quality of roads, airports, etc.). Therefore, further analyses are needed to evaluate how transportation presents as barrier. It is possible that wealth may modify the association between distance and compliance. Specifically, more affluent people may be less sensitive to distance as a barrier in that they can afford to travel easily. Unfortunately, as individual level income data were not available, we could not address this possibility. Finally, while our study assessed the negative impact of 
regionalization, due to the limits of the data, we are unable to assess potential benefits of regionalization (i.e., improved quality of care) or examine survival or cost-benefit analyses. Further studies are needed to examine the net benefits vs. risks of regionalization, particularly for other diseases and countries.

Despite these limitations, our study has several key strengths. Given the high level of illiteracy, investigators contacted men whose biopsy indications were suspicious of PC by mail and by phone. Including a phone call as a notification method ensured that the men received the information and were able to understand it. If notifications were only by mail, this could be a major explanatory variable for the low follow-up rates in illiterate men. Secondly, contamination issues commonly seen in large-scale screening studies were minimized as we restricted analyses to first screens. Thirdly, there were standardized methods of data collection for each enrolled subject with over $94 \%$ of men having complete data. The fact that all data were collected prior to patients knowing their screening results minimized recall bias. The same personnel performed DRE exams throughout the study period. This ensured uniformity in classification of PC risk. Regarding postscreen biopsies, there was limited variability in the number of biopsy cores taken (median: 12, IQR: 10-14), consistent with global standards to optimize PC detection [22]. Most notably, when screening programs are instituted, it is important to ensure that there are provisions for people with positive screens to be able to receive appropriate follow-up and care. With such plans in place, our study is comprised of data from a large cohort of rural Brazilian men, who have previously not been well-studied, providing unique insights into an understudied population.

\section{Conclusion}

Among Brazilian men undergoing initial PC screening, we found greater distance from local screening site to regional follow-up care facility was a significant risk factor for noncompliance to biopsy recommendations. However, when distances were $<150 \mathrm{~km}$, distance was unrelated to biopsy compliance. Finally, we found no association between distance and risk of PC or PC grade on biopsy among those who underwent a biopsy. While regionalization of care may in theory improve quality, it comes at the cost of reduced compliance and reduced access and represents a significant barrier to optimal care if distances are large.

\section{Abbreviations}

BCH: Barretos Cancer Hospital

LOWESS: Locally weighted smoothed scatterplot

MCPSU: Mobile Cancer Prevention Screening Unit.

\section{Data Availability}

The data used to support the findings of this study are restricted by the IRB of Barretos Cancer Hospital in order to protect patient privacy.

\section{Ethical Approval}

This research study was conducted retrospectively from data obtained for clinical purposes. We consulted extensively with the Institutional Review Board (IRB) of Barretos Cancer Hospital who determined that our study did not need ethical approval. An IRB official waiver of ethical approval was granted from the IRB of Cedars-Sinai Medical Center.

\section{Consent}

Informed consent was obtained from all individual participants included in the study. The authors affirm that human research participants provided informed consent for publication.

\section{Disclosure}

This work has been previously presented at the American Urological Association annual meeting (2017).

\section{Conflicts of Interest}

The authors have no conflicts of interest to disclose.

\section{Authors' Contributions}

AR Freedland and SJ Freedland contributed to project development, data analysis, and manuscript writing/editing. RL Muller contributed to project development and manuscript editing. C Hoyo, EL Turner, and PG Moorman contributed to data analysis and manuscript editing. EF Faria contributed to protocol development and data collection/management. GF Carvalhal, RB Reis, EC Mauad, and AL Carvalho contributed to protocol development.

\section{Acknowledgments}

Barretos Cancer Hospital and Pio XII Foundation sponsored the screening program. This work was supported by the United States Department of Defense (W81XWH-10-10155).

\section{References}

[1] G. A. E. Silva, C. J. Gamarra, V. R. Girianelli, and J. G. Valente, "Cancer mortality trends in Brazilian state capitals and other municipalities between 1980 and 2006," Revista de Saúde Pública, vol. 45, no. 6, pp. 1009-1018, 2011.

[2] P. Kanavos, "The rising burden of cancer in the developing world," Annals of Oncology, vol. 17, 2006.

[3] A. L. Potosky, "The role of increasing detection in the rising incidence of prostate cancer," JAMA: The Journal of the American Medical Association, vol. 273, no. 7, pp. 548-552, 1995.

[4] M. W. Vetterlein, B. Löppenberg, P. Karabon et al., "Impact of travel distance to the treatment facility on overall mortality in US patients with prostate cancer," Cancer, vol. 123, no. 17, pp. 3241-3252, 2017. 
[5] S. R. G. Finlayson, J. D. Birkmeyer, A. N. A. Tosteson, and R. F. Nease, "Patient preferences for location of care," Medical Care, vol. 37, no. 2, pp. 204-209, 1999.

[6] J. J. Guidry, L. A. Aday, D. Zhang, and R. J. Winn, “Transportation as a barrier to cancer treatment," Cancer Practice, vol. 5, no. 6, pp. 361-366, 1997.

[7] M. J. Wills, M. V. Whitman, and T. M. English, "Travel distance to cancer treatment facilities in the deep South," Journal of Healthcare Management, vol. 62, no. 1, pp. 30-43, 2017.

[8] J. M. Singh and R. D. MacDonald, "Pro/con debate: do the benefits of regionalized critical care delivery outweigh the risks of interfacility patient transport?" Critical Care, vol. 13, no. 4, p. 219, 2009.

[9] C. Kelly, C. Hulme, T. Farragher, and G. Clarke, "Are differences in travel time or distance to healthcare for adults in global north countries associated with an impact on health outcomes? A systematic review," BMJ Open, vol. 6, no. 11, Article ID e013059, 2016.

[10] O. O'Donnell, "Access to health care in developing countries: breaking down demand side barriers," Cadernos de Saúde Pública, vol. 23, no. 12, pp. 2820-2834, 2007.

[11] E. F. Faria, G. F. Carvalhal, R. A. C. Vieira, T. B. Silva, E. C. Mauad, and A. L. Carvalho, "Program for prostate cancer screening using a mobile unit: results from Brazil," Urology, vol. 76, no. 5, pp. 1052-1057, 2010.

[12] R. L. Muller, E. F. Faria, G. F. Carvalhal et al., "Association between family history of prostate cancer and positive biopsies in a Brazilian screening program," World Journal of Urology, vol. 31, no. 5, pp. 1273-1278, 2013.

[13] P. M. Pierorazio, P. C. Walsh, A. W. Partin, and J. I. Epstein, "Prognostic Gleason grade grouping: data based on the modified Gleason scoring system," BJU International, vol. 111, no. 5, pp. 753-760, 2013.

[14] R. W. Dobbs, N. R. Malhotra, B. M. Caldwell, R. Rojas, D. M. Moreira, and M. R. Abern, "Determinants of clinic absenteeism: a novel method of examining distance from clinic and transportation," Journal of Community Health, vol. 43, no. 1, pp. 19-26, 2018.

[15] J. A. Holmes, W. R. Carpenter, Y. Wu et al., "Impact of distance to a urologist on early diagnosis of prostate cancer among black and white patients," Journal of Urology, vol. 187, no. 3, pp. 883-888, 2012.

[16] P. M. Marcus, S. L. Ogden, L. H. Gren et al., "Non-compliance with the initial screening exam visit in the prostate, lung, colorectal and ovarian (PLCO) cancer screening trial," Preventive Medicine, vol. 67, pp. 82-88, 2014.

[17] J. F. Scoggins, C. R. Fedorenko, S. M. A. Donahue, D. Buchwald, D. K. Blough, and S. D. Ramsey, "Is distance to provider a barrier to care for medicaid patients with breast, colorectal, or lung cancer?" The Journal of Rural Health, vol. 28, no. 1, pp. 54-62, 2012.

[18] M. Ambroggi, C. Biasini, C. Del Giovane, F. Fornari, and L. Cavanna, "Distance as a barrier to cancer diagnosis and treatment: review of the literature," The Oncologist, vol. 20, no. 12, pp. 1378-1385, 2015.

[19] G. F. Nemet and A. J. Bailey, "Distance and health care utilization among the rural elderly," Social Science \& Medicine, vol. 50, no. 9, pp. 1197-1208, 2000.

[20] M. A. Parsons and K. D. Askland, "Cancer of the colorectum in Maine, 1995-1998: determinants of stage at diagnosis in a rural state," The Journal of Rural Health, vol. 23, no. 1, pp. 25-32, 2007.
[21] M. D. Silverstein, P. J. Nietert, X. Ye, and D. T. Lackland, "Access to care and stage at diagnosis for patients with lung cancer and esophageal cancer," Southern Medical Journal, vol. 95, no. 8, pp. 900-908, 2002.

[22] A. R. Patel and J. S. Jones, "Optimal biopsy strategies for the diagnosis and staging of prostate cancer," Current Opinion in Urology, vol. 19, no. 3, pp. 232-237, 2009. 